\title{
Role of Interface Structure and Chemistry in Resistive Switching of NiO Nanocrystals on $\mathrm{SrTiO}_{3}$
}

\author{
Xuan Cheng ${ }^{1}$, Jivika Sullaphen ${ }^{1}$, Matthew Weyland ${ }^{2,3}$, Hongwei Liu ${ }^{4}$, and Nagarajan Valanoor ${ }^{1}$ \\ 1. School of Materials Science and Engineering, UNSW Australia, Sydney, Australia \\ 2. Department of Materials Engineering, Monash University, Melbourne, Australia \\ 3. Monash Centre for Electron Microscopy, Monash University, Melbourne, Australia \\ 4. Australian Centre for Microscopy and Microanalysis, University of Sydney, Sydney, Australia
}

Nickel oxide $(\mathrm{NiO})$ is a binary metal oxide that is found to have resistive switching (RS) properties, which makes it a viable candidate for next generation resistive random access memories (RRAMs). The demand for high-density memories has concentrated on RS materials with scalable dimensions. In these nanoscaled devices, the presence of point and line defects, composition inhomogeneity, and atomic interdiffusion interfaces have been shown to have a significant impact on properties.

The objective of this study is to investigate the role of interface morphology and chemistry in resistive switching behavior of $\mathrm{NiO}$ epitaxial nanocrystals fabricated on (001) $\mathrm{SrTiO}_{3}$ (STO) substrates [1]. NiO nanostructures are found to have $\{111\}$ and $\{113\}$ orientated facets. The epitaxial relationship between the nanostructure and the substrate can be confirmed. The crystallographic orientations of the nanostructures are $[110]_{\mathrm{NiO}} / /[110]_{\mathrm{STO}}$ inplane and $[100]_{\mathrm{NiO}} / /[100]_{\mathrm{STO}}$ out of plane, confirming that the nanostructure and substrate are aligned in a cubic-on-cubic fashion.

Aberration corrected high angle annular dark field (HAADF) scanning transmission electron microscopy (STEM) reveals the interface between the $\mathrm{NiO}$ nanocrystals and the underlying STO substrate to be rough, irregular, and have a lower average atomic number than the substrate or the nanocrystal [1]. Energy dispersive x-ray spectroscopy and electron energy loss spectroscopy (shown in Figure 1) confirm both chemical disorder and a shift of the energy of the Ti $L_{2,3}$ peaks. Analysis of the $\mathrm{O} K$-edge profiles in conjunction with this shift, implies the presence of oxygen vacancies at the interface. This sheds light into the origin of the previously postulated minority carriers' model to explain resistive switching in $\mathrm{NiO}[2]$.

HRTEM and HAADF-STEM reveal that the substrate is deformed (see Figure 2) so as to back-fill around the base of the nanostructure in order to promote wetting. Remarkably, the substrate under the nanocrystal is physically pulled towards the nanostructure, thus forming a rim around the nanocrystal [4].

\section{References:}

[1] X. Cheng et al, Appl. Phys. Lett. Mater. 2 (2014), p. 032109

[2] J. Sullaphen et al, Appl. Phys. Lett.100 (2012), p. 203115

[3] The research at UNSW was supported by an ARC Discovery and LIEF Grant. The research at Monash Centre for Electron Microscopy (MCEM) used equipment funded by Australian Research Council Grant No. LE0454166 (FEI Titan 80-300 FEGTEM). We thank MCEM, Microscopy Units (EMU) at UNSW and Australian Centre for Microscopy and Microanalysis (ACMM) at USYD for the provision of equipment and technical support. The author would also like to acknowledge Dr. Ye Zhu 
for his contribution in discussing the results.
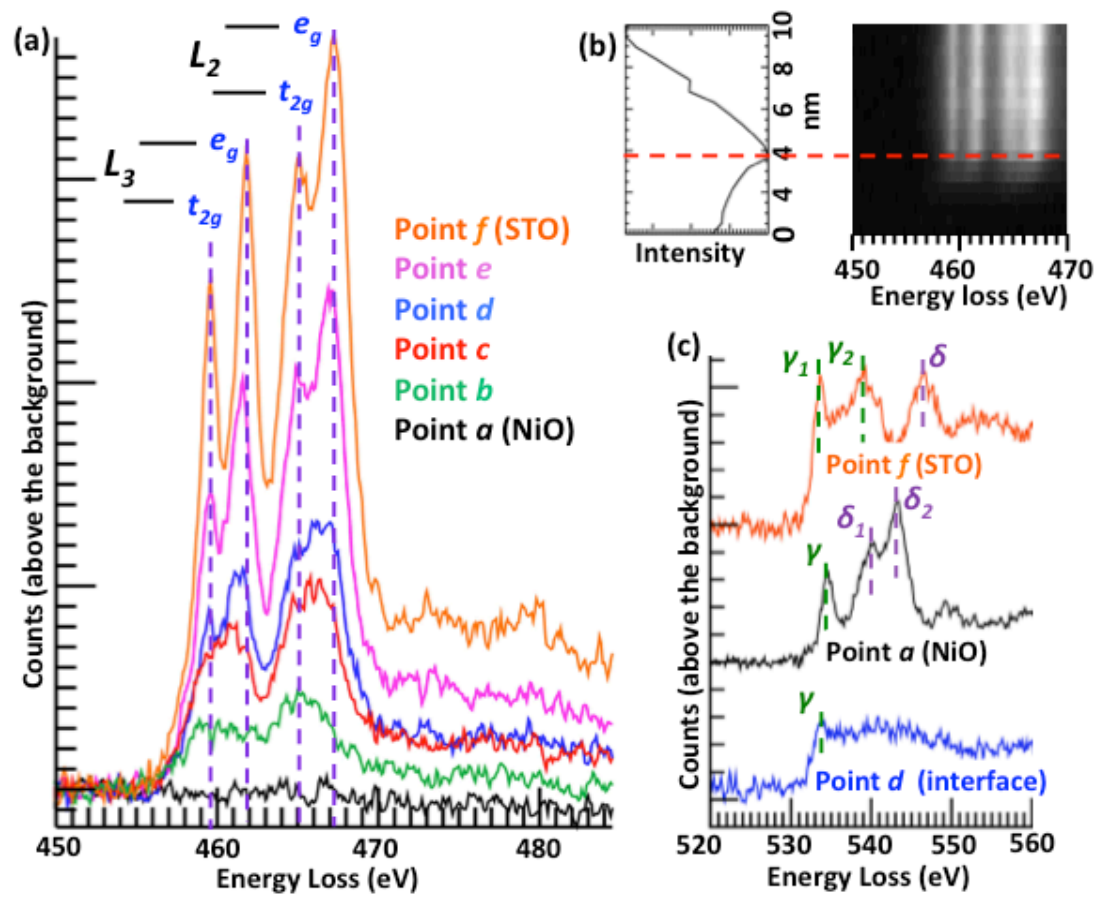

Figure 1. (a) Ti $L$ edges of the selected points from EELS line scan. (b) ADF intensity plot and EEL spectrum image simultaneously acquired with EELS. (c) Simultaneously acquired $\mathrm{O} K$ edges from point $a, d$, and $f$.

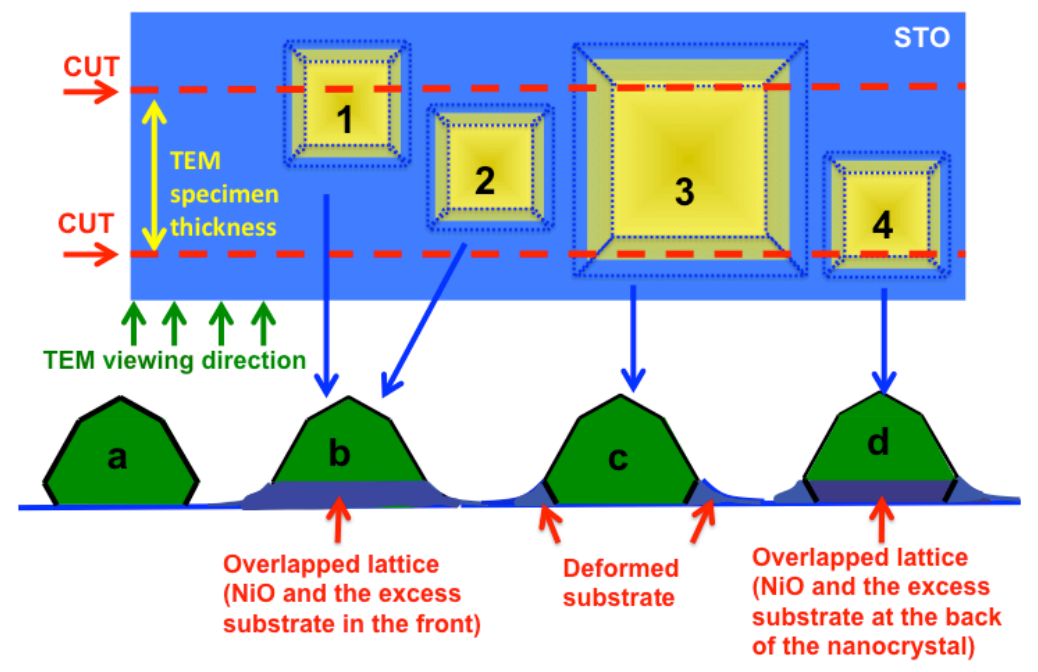

Figure 2. Schematic of top view image (top) and cross-sectional transmission electron microscope (TEM) image (bottom) shows different appearance of substrate in TEM image due to the cut position of $\mathrm{NiO}$ in TEM sample preparation. In the top-view image, $\mathrm{NiO}$ nanocrystals, the substrate, and the overlapped area due to the substrate deformation are shown as yellow, blue and green colour respectively. The deformed substrate around $\mathrm{NiO}$ is indicated with blue dotted lines 